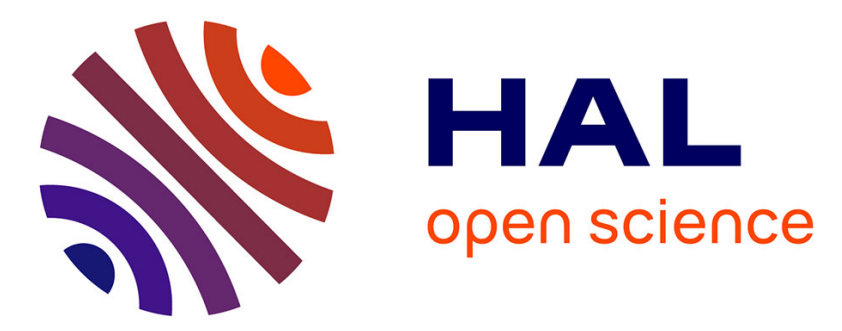

\title{
Effect of fiber Bragg Gratings receivers' directivity on guided wave tomography of pipe
}

\author{
Tom Druet, Huu Tinh Hoang, Simon Nehr, Guillaume Laffont, Bastien
}

Chapuis

\section{- To cite this version:}

Tom Druet, Huu Tinh Hoang, Simon Nehr, Guillaume Laffont, Bastien Chapuis. Effect of fiber Bragg Gratings receivers' directivity on guided wave tomography of pipe. IWSHM 2019 - The 12th International Workshop on Structural Health Monitoring, Sep 2019, Stanford, United States. $10.12783 / \mathrm{shm} 2019 / 32191$. cea-03252957

\section{HAL Id: cea-03252957 https://hal-cea.archives-ouvertes.fr/cea-03252957}

Submitted on 8 Jun 2021

HAL is a multi-disciplinary open access archive for the deposit and dissemination of scientific research documents, whether they are published or not. The documents may come from teaching and research institutions in France or abroad, or from public or private research centers.
L'archive ouverte pluridisciplinaire HAL, est destinée au dépôt et à la diffusion de documents scientifiques de niveau recherche, publiés ou non, émanant des établissements d'enseignement et de recherche français ou étrangers, des laboratoires publics ou privés. 
Title: Effect of Fiber Bragg Gratings receivers' directivity on Guided Wave Tomography of pipe

\author{
Authors : Tom Druet \\ Huu Tinh Hoang \\ Simon Nehr \\ Guillaume Laffont \\ Bastien Chapuis
}




\begin{abstract}
Structural health monitoring (SHM) consists in embedding sensors in a structure like aircraft fuselages, pipes or ship hulls in order to detect defects (for example cracks or corrosion in metallic materials or delamination in composite materials) before a serious fault occurs in the structure. Guided elastic waves emitted by a sensor and propagating to another one are often used as the physical way of detecting the defect. However, the implementation of SHM systems is restricted in many situations by the necessity to store or to harvest the electric energy necessary to emit the waves and also by the intrusiveness of the sensors. Guided wave tomography imaging is able to localize and quantify the severity of the defect when it comes to loss of thickness such as corrosion or erosion. However, it needs many sensors (generally piezoelectric - PZT - transducers) and it has a cost, particularly in terms of intrusiveness. The idea in this paper is to use less intrusive sensors such as fiber Bragg Gratings (FBG) to perform guided wave tomography. The inspected area of a pipe is surrounded by two rings, one with PZT and the other one with FBG.
\end{abstract}

\title{
INTRODUCTION
}

Structural Health Monitoring (SHM) consists of acquiring and analyzing data from sensors embedded in a structure in order to evaluate its health [1]. A promising technology relies on the use of piezoelectric transducers emitting and receiving guided waves in the structure. However, this approach becomes very intrusive in combination with imaging algorithms such as tomography due to the large number of sensors required [2].

An interesting possibility is to design a guided wave based SHM system with minimal intrusiveness thanks to the use of fiber Bragg Gratings (FBG) sensors on optical fibers. However, these sensors cannot emit waves, they can only work in a passive way. This means that passive methods are needed to retrieve the response between two sensors. In the past years, researchers have shown that it is possible to use ambient structure-borne elastic noise instead of actively emitting the waves in the structure in order to retrieve the impulse response of the medium between two sensors [3]. The idea

Tom Druet, Huu Tinh Hoang, Simon Nehr, Guillaume Laffont, Bastien Chapuis, CEA, LIST, F-91191 Gif-sur-Yvette, France. Email: tom.druet@cea.fr 
is to take advantage of the elastic noise naturally present in the structure in order to avoid the emission of the elastic waves by the SHM system. The complexity of the embedded SHM system is therefore reduced.

Passive guided wave tomography using piezoelectric transducers has been studied in [4] for plate structures and with a noise generated by a jet of compressed air. This method is transposed for pipe structures in [5]. It has also be shown in [6] that passive guided waves measurements using FBG sensors is possible thanks to passive methods such as the ambient noise cross-correlation. In this paper the studies are focused on the impacts that have the fiber Bragg Gratings measurements on guided wave tomography imaging on pipe structure because of the FBG directivity. In a first part, the diffraction tomography algorithm used in this paper is briefly described. Then, the effect of FBG directivity on guided waves signal measurements and tomography resolution are highlighted.

\section{METHODS}

In this first section the method which allows to obtain and to interpret the results of the following section is presented. Firstly, the imaging algorithm used is introduced and then the directional measurement of the fiber Bragg Grating is presented.

\section{Guided Wave Tomography}

Guided elastic wave tomography is a method giving a quantitative image of an inspected area with potential corrosion or erosion flaws. This imaging method is very interesting because it can be baseline-free (see [2]) and so reduces the risks of false alarms for a SHM system.

In this paper is used classical diffraction tomography (DT) algorithm [7] on pipe structure. The image is represented by an object function $\mathrm{O}$ at the position $\boldsymbol{x}$ :

$$
\mathrm{O}(\boldsymbol{x})=k(\boldsymbol{x})^{2}-k_{0}^{2}=k_{0}^{2}\left(\left(\frac{v_{0}}{v(\boldsymbol{x})}\right)^{2}-1\right),
$$

where $k(\boldsymbol{x})$ is the wavenumber at the position $\boldsymbol{x}, k_{0}$ the background wavenumber, $v_{0}$ the background phase velocity and $v(\boldsymbol{x})$ the phase velocity at the position $\boldsymbol{x}$.

Diffraction tomography allows to solve the inverse problem to calculate the object function:

$$
\mathrm{O}(\boldsymbol{x})=\int_{-\pi}^{\pi} \int_{-\pi}^{\pi} \frac{\hat{\varphi}_{\boldsymbol{k}_{0}}^{s}\left(\boldsymbol{x}_{l}\right)}{G_{0}\left(\boldsymbol{x}_{l} ; \boldsymbol{x}\right) G_{0}\left(\boldsymbol{x} ; \boldsymbol{x}_{k}\right)} \mathrm{W}\left(\theta_{k}, \theta_{l}\right) \mathrm{d} \theta_{k} \mathrm{~d} \theta_{l},
$$

where $\hat{\varphi}_{\boldsymbol{k}_{0}}^{s}\left(\boldsymbol{x}_{l}\right)$ is the scattered field measured at the position $\boldsymbol{x}_{l}$ by the receiver number $l$, $G_{0}$ the non-perturbed Green's function, $k$ the number of the emitter and W a weighting function which comes from a variables' change between spatial frequencies and angular coordinates. The link between the object function and the thickness is then given by the dispersion curves. More details about the algorithm used here are given in [5].

\section{Fiber Bragg Gratings}




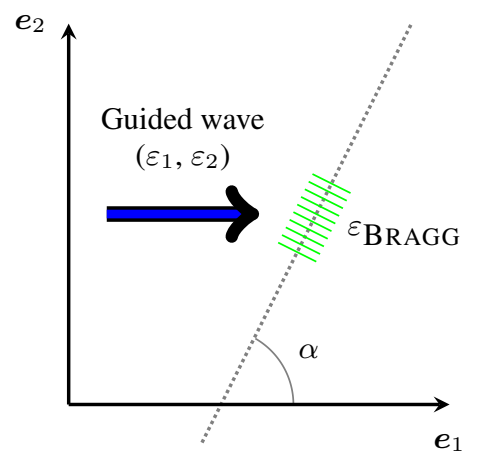

Figure 1: Fiber Bragg Gratings’ parameters.

It is well known that fiber Bragg Gratings make directional measures. For isotropic structures, this sensitivity reads [8]:

$$
\varepsilon_{\mathrm{BRAGG}}=\varepsilon_{1} \cos ^{2}(\alpha)+\varepsilon_{2} \sin ^{2}(\alpha),
$$

where $\varepsilon_{\text {BRAGG }}$ is the strain of the sensor, $\varepsilon_{1}$ is the strain due to the wave in the direction $\boldsymbol{e}_{1}, \varepsilon_{2}$ is the strain in the direction $\boldsymbol{e}_{2}$ and $\alpha$ is the incident angle of the wave with respect to the FBG's axis (see the Figure 1).

Effects on the measured signals are presented in the following section.

\section{EXPERIMENTAL RESULTS}

First, this section presents experimental signals measured with fiber Bragg gratings. The effect of the directivity is evaluated by comparing those signals with piezoelectric measurements. Then, hybrid guided wave tomography of erosion in pipe is presented. One ring of piezoelectric transducers is used to emit guided waves and an other one made up of fiber Bragg gratings is used to measure the waves.

\section{Effects of the Directivity}

In this section the sensitivity of the fiber Bragg gratings with respect to the angle between the FBG's axis and the direction of the wave propagation is presented. For this purpose, on FBG was glued on an $2 \mathrm{~mm}$ aluminum plate. In order to emit a guided wave one PZT generated a toneburst for several angles between the FBG's axis and the PZT. Some of the signals measured by the FBG are presented in Figure 2(a). It can be noticed that the signal is maximal for $0^{\circ}$ and minimal for $90^{\circ}$. The energy of the wave packets is then compared to the theoretical curve of Equation 3 in Figure 2(b). The experimental curve fits quite well the theoretical one.

Now that the theoretical directivity curve is verified on a plate, the pipe geometry is considered. Two rings of sixteen PZT for the first one and sixteen FBG for the second one were glued on a pipe of stainless steel. The rings are separated by a distance of $40 \mathrm{~cm}$. A flaw was created in the inspection area by electrolysis. The experimental setup is presented in Figure 3.

An acquisition was performed before the flaw's creation. The signals measured by 


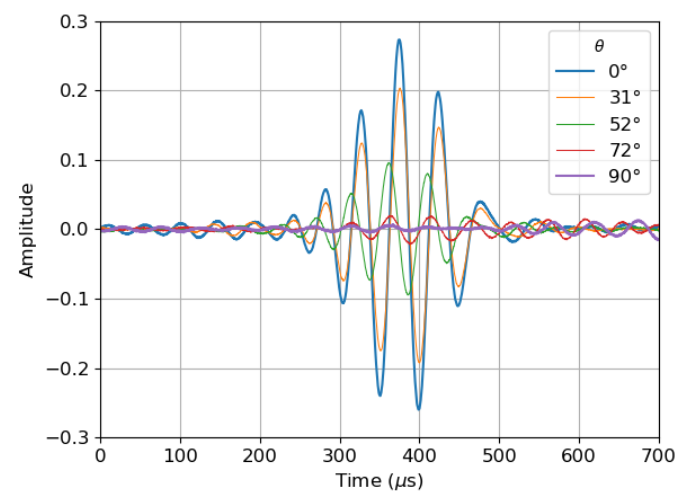

(a)

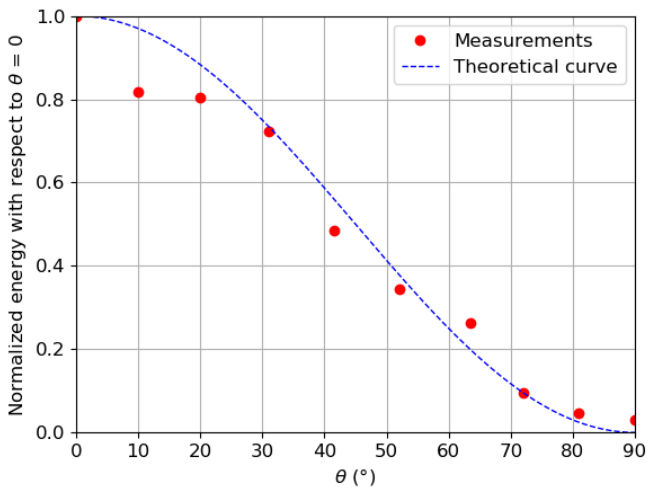

(b)

Figure 2: Fiber Bragg Gratings' directivity. (a) Signals measured by FBG on a plate. (b) Experimental and theoretical comparison of the directivity.

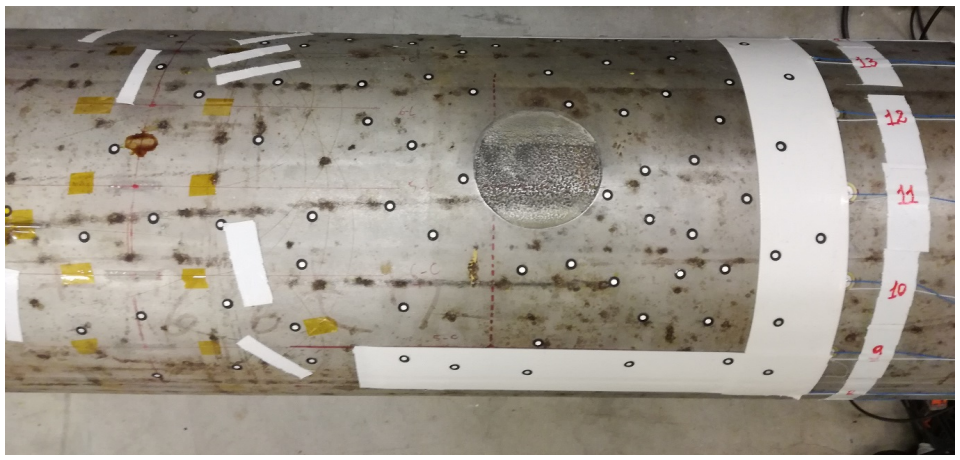

Figure 3: Experimental setup.

the FBG ring for one PZT emission are presented in Figure 4(b). To see the effect of the directivity the same acquisition was performed but with a ring of fifteen PZT transducers. The signals are presented in Figure 4(a). For the PZT case (Figure 4(a)), both the direct waves (first arrivals) and the helical waves have the same amplitude for all the signals. This is due to the omni-directional measurement of the PZT transducers. However, for the FBG's case (Figure 4(b)), the more the angle between the FBG's axis and the direction of propagation is high, the more the signal measured by the FBG is small. That phenomenon is even more pronounced for the helical paths where the wave packets are difficult to distinguish. This could become an issue when the helical modes are used in the guided wave tomography process to increase the lateral resolution due to the lack of sensors.

\section{Imaging with Piezoelectric Transducers and Fiber Bragg Gratings}

In this section hybrid guided wave tomography of a $2.145 \mathrm{~mm}$ thick pipe of stainless steel is presented. Its diameter is $250 \mathrm{~mm}$. The experimental setup is recalled in Figure 3 where it is possible to see an erosion flaw created by an electrolysis process. This flaw is first imaged thanks to a methods much more precise than guided wave tomography. 


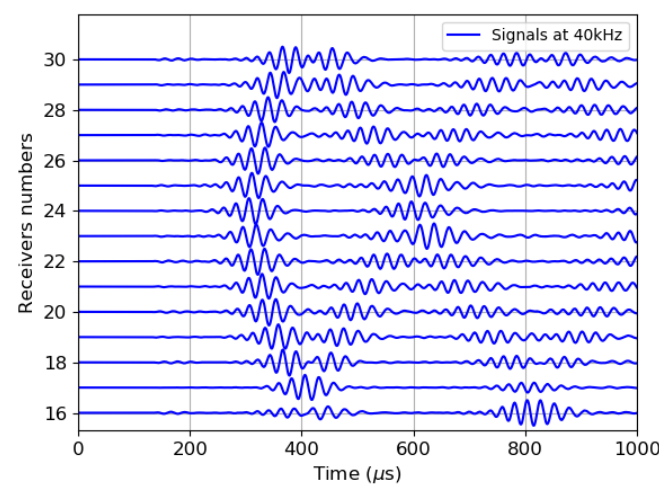

(a)

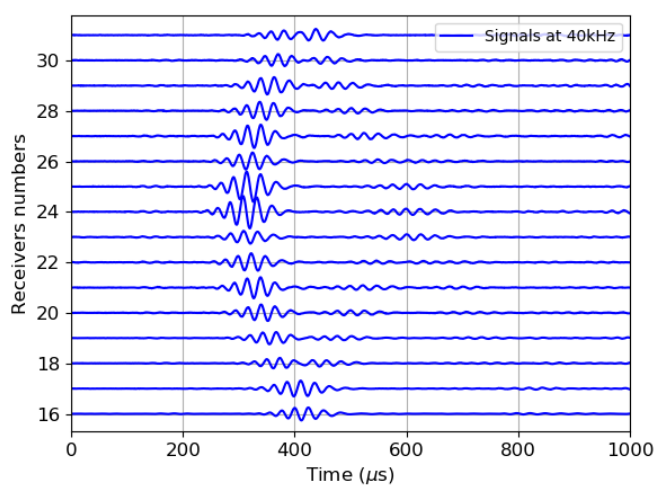

(b)

Figure 4: Effect of the fiber Bragg gratings' directivity compared to piezoelectric transducers. (a) PZT. (b) FBG.

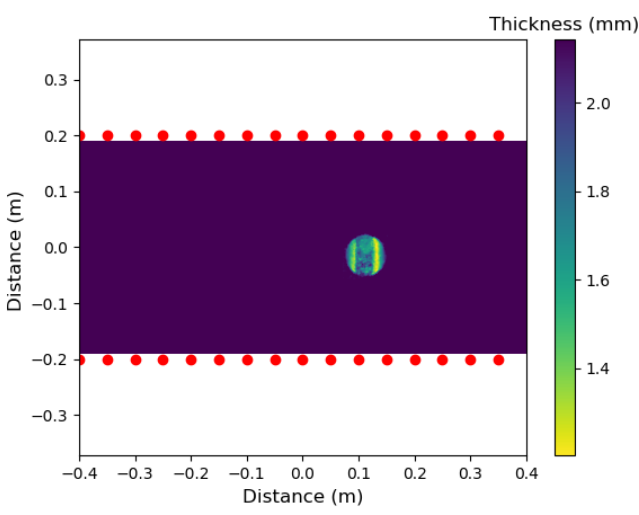

(a)

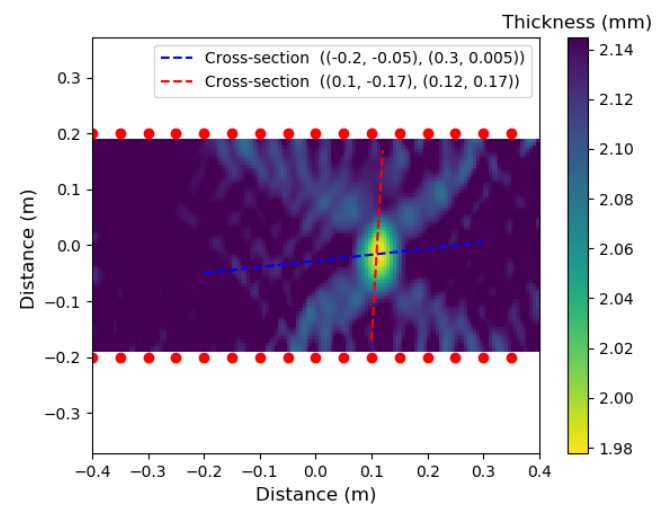

(b)

Figure 5: Hybrid guided wave tomography of the erosion flaw of Figure 3. (a) Reference measured with a 3D scanner. (b) Image obtained with guided wave tomography.

Indeed, a reference of the erosion flaw is measured by a system called GoScan which is able to performed a 3D scan. This reference is shown in Figure 5(a).

In order to performed the hybrid guided wave tomography, the PZT emitters ring generated alternatively a five-cycles Hann windowed toneburst centered from $20 \mathrm{kHz}$ to $60 \mathrm{kHz}$ and the measurements was made by the ring of FBG receivers. The result of the diffraction tomography - the mean between the images computed for all the frequencies - is presented in Figure 5(b). The erosion defect is well localized but the small spatial variations are not identified because of the resolution limit of the algorithm directly linked to the wavelength being about $20 \mathrm{~mm}$, depending on the frequencies. Moreover, this is an undersampled data case which further restricts the resolution [2].

In order to be able to assess the quality of the reconstruction, two cross-sections are plotted in Figure 6. The blue one - Figure 6(a) - is lateral (see Figure 5(b)) and the red one - Figure 6(b) - is axial (see Figure 5(b)). As expected the lateral resolution is better than the axial one because of the lack of sensors along the pipe's axis. Moreover, 


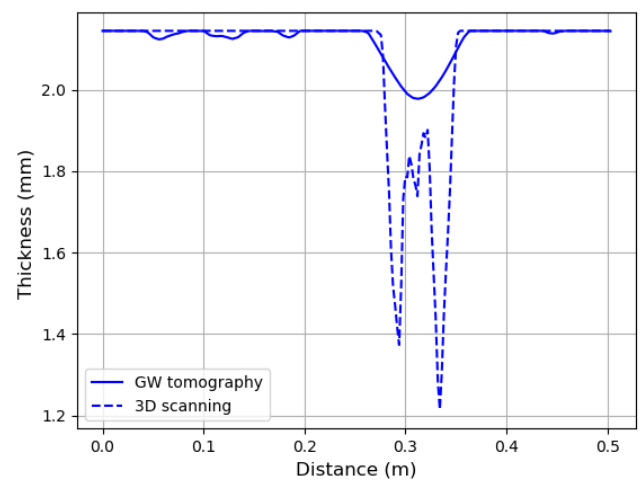

(a)

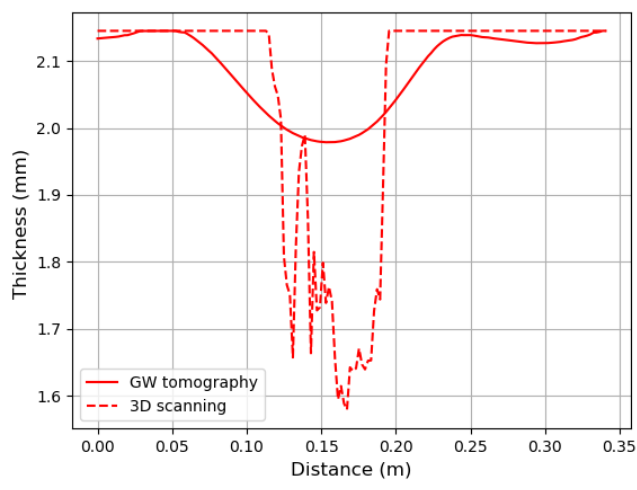

(b)

Figure 6: Cross-sections of the Figure 5. (a) Blue lateral cross-section. (b) Red axial cross-section.

once more it is possible to see that the small spatial variation are not identified because of the resolution limit. Finally, the real depth of the flaw is not reached compared to classical PZT/PZT guided wave tomography (see [5]). This is due to two phenomena. The first one is because only one ring is used as emitter. Indeed, fiber Bragg gratings cannot emit waves and so only half the data is available. The second phenomenon is the directivity, it has been shown above that the signals measured by the FBG are smaller than the signals measured by the PZT (see Figure 4). This phenomenon has a direct impact on the amplitude of the field used for the tomography and so a direct impact on the final reconstruction.

There is still work to do about these two phenomena in order to improve the imaging process when directional sensors like FBG are used and this will be the purpose of future works.

\section{CONCLUDING REMARKS}

In this paper, the impact of the fiber Bragg gratings directivity on guided wave tomography has been studied. For this work, it has been decided to perform active guided wave tomography by using one ring of piezoelectric transducers and one ring of fiber Bragg grating sensors glued on the pipe to inspect. The image obtained is almost of same quality that classical PZT/PZT guided wave tomography (see for instance [5]) but there is still an effect of the directivity on the depth reconstruction. Indeed, this directivity involves a bias on the reconstruction because of the variation of the signals' amplitude depending on the angle between the FBG's axis and the direction of the wave propagation. Studies could be done to compensate the directivity effects in the near future.

A long-term goal is to perform passive guided wave tomography only with fiber Bragg gratings sensors in order to be able to design a guided wave based SHM system with minimal intrusiveness. 


\section{REFERENCES}

1. 2013. "Guidelines for Implementation of Structural Health Monitoring on Fixed Wing Aircraft," SAE International, ARP6461.

2. Druet, T., J.-L. Tastet, B. Chapuis, and E. Moulin. 2019. "Autocalibration method for guided wave tomography with undersampled data," Wave Motion, 89:265 - 283, ISSN 0165-2125, doi:https://doi.org/10.1016/j.wavemoti.2019.04.002.

3. Weaver, R. and O. Lobkis. 2001. "Ultrasonics without a Source: Thermal Fluctuation Correlations at MHz Frequencies," Physical Review Letters, 87(13):134301.

4. Druet, T. 2017. Tomographie passive par ondes guidées pour des applications de contrôle santé intégré, Ph.D. thesis, Université de Valenciennes et du Hainaut-Cambresis.

5. Hoang, H., T. Druet, B. Chapuis, and E. Moulin. 2019. "Passive Tomography by Elastic Guided Wave for Corrosion Detection in Pipelines," in Proceedings of the 12th International Workshop on Structural Health Monitoring, Stanford.

6. Druet, T., B. Chapuis, M. Jules, G. Laffont, and E. Moulin. 2018. "Passive guided waves measurements using fiber Bragg gratings sensors," The Journal of the Acoustical Society of America, 144(3):1198-1202, doi:10.1121/1.5054015.

7. Huthwaite, P. 2016. "Eliminating incident subtraction in diffraction tomography," Proceedings of the Royal Society of London A: Mathematical, Physical and Engineering Sciences, 472(2195), ISSN 1364-5021, doi:10.1098/rspa.2016.0707.

8. Betz, D., G. Thursby, B. Culshaw, and W. Staszewski. 2007. "Structural Damage Location with Fiber Bragg Grating Rosettes and Lamb Waves," Structural Health Monitoring, 6:299_ 308. 